\title{
Amish Settlements across America: 2013
}

\author{
Joseph F. Donnermeyer ${ }^{1}$ \\ Professor, Rural Sociology Program \\ School of Environment and Natural Resources \\ The Ohio State University \\ Columbus, Ohio 43210 \\ David Luthy \\ Heritage Historical Library \\ 52445 Glencolin Line \\ Aylmer, Ontario N5H 2R3
}

\begin{abstract}
This short research report is based upon previous editions of "Amish Settlements across North America," which was published periodically in Family Life. It accounts for new settlements founded since the last edition (2008), as well as settlements which are recently extinct. The information is presented in a series of six tables, including a list of all Amish settlements as of September 30, 2013 (Table 1). Table 2 summarizes the number of settlements and church districts in each state, while Tables 3 and 4 shows trends in settlement increases, decade by decade, since 1900. Table 5 is a list of settlements which became extinct between 2009 and September 30, 2013. Finally, Table 6 describes 15 facts about Amish settlements - past and present - plus, a projection about future settlement growth. We include a map showing the geographic distribution of settlements across Canada and the United States.
\end{abstract}

\section{Keywords}

doubling time, settlement, settlement growth, Amish

\section{Acknowledgements}

We acknowledge the work of Loren Kenda, a graduate student in the College of Education and Human Ecology, The Ohio State University, on production of the map for this article. 
The last published directory of Amish settlements included all known communities, as of December 2008 (Luthy 2009). At that time, there were 402 settlements and 1,735 church districts. This new list shows 472 Amish settlements and 2,012 church districts through September 2013. In the short period of four years and nine months-45 months in all-there has been a net increase of 70 settlements and 277 church districts.

The increase in the number of church districts reflects the continual growth of the Amish population. The increase in the number of settlements reflects the need for affordable land / real estate to accommodate the rising population. One estimate places the current doubling time (i.e., the time it takes a population to double in size) of the Amish at 21.25 years (Donnermeyer and Cooksey 2010). Family size and the high percentage of daughters and sons who decide to be baptized in the Amish faith when they reach the age of decision are the two most immediate factors that explain this growth (Donnermeyer and Cooksey 2010). However, behind these two facts are more fundamental reasons associated with religious values and the gulf between mainstream North American society and the ways Amish church and community are organized.

For this article, we maintain the same definition of an Amish settlement used in previous editions of the Amish Settlements across America series, printed and distributed by Pathway Publishers:

To be included in this directory, a new settlement must initially have at least three resident households-or two, if one household head is in the ministry. Each new settlement will either soon grow or falter and disband. Formerly active settlements are not included if church services are no longer held, even though a few families or members may still reside there (Luthy 2009, 1).

Although as little as two or three families may seem to some to hardly constitute an active settlement, the definition has a decided advantage. There is little room for interpretation when the number of households is set at so low a threshold, because the real focus should not be on numbers, but on the ability of a fledging community to hold a church service. For example, would five families or 10 families be better cut-offs? We think not! Also maintained are two other features from previous editions of Amish Settlements across America:

...to be included in this directory, the settlement must forbid ownership of motor vehicles and use the name 'Amish'. Thus, included are not merely standard Old Order Amish settlements, but New Order Amish, Swartzentruber Amish, Troyer Amish, Nebraska Amish, and even small splinter groups. The only time that distinctions are made in the directory's state-by-state listing is when two types of Amish establish separate settlements at the same location (Luthy 2009, 2).

However, we do not include groups considered to be “para-Amish” (Waldrep 2008) ${ }^{2}$ such as the plain communities at Bergholz, Ohio; Caneyville and Hestand, Kentucky; and Lobelville, Tennessee. 
Finally, we adhere to the same conservative approach to the list of Amish communities adopted in previous editions of Amish Settlements across America. Again, to quote:

Caution should always be used before declaring a shrinking settlement as extinct. Undoubtedly, there are a few in this directory which may be nearing extinction, but a 'wait and see' policy is always best. Caution must also be used to avoid including a new settlement which actually does not qualify. Such a mistake occurred in the 2003 directory when Oakfield, Wisconsin was shown as having been founded in 1998. It was included because the Kingston, Wisconsin scribe referred to it as 'a new settlement.' When later contacted about its existence, he said that only one family had ever moved there. He explained that 'more had plans, but they never materialized.' The one family there soon moved back to the Kingston community. Thus, Oakfield never really was a settlement. Nor was Hiseville, Kentucky which also was mistakenly included in the 2003 directory (Luthy 2009, 2).

Included in this report are six tables that summarize both past and recent developments in the spread of Amish settlements throughout North America.

Table 1 is a list of existing settlements, state by state, including the year a community was founded and the estimated number of church districts associated with each settlement. For church district estimates, we relied on two primary sources. One was reports from scribes about "statistics for this community" as found in The Budget, Die Botschaft, and The Diary. The other was the most recent edition of The New American Almanac 2013 (Raber 2012), which includes a list of church leaders for most, but not all, church districts.

Communities range from as far east as Fort Fairfield, Maine, and as far south as Pinecraft, Florida. The settlement near Rexford, Montana, has a dual distinction. It is the northernmost Amish settlement, with a latitude greater than both the Englehart or Earlton settlements in Ontario. It is also the westernmost Amish community, with a longitude greater than the recently founded settlement near Salmon, Idaho.

Displayed in Table 2 is a summary of Amish settlements, along with the number of church districts for each state / province. Altogether, Amish settlements can be found in 30 U.S. states and the Canadian province of Ontario. Pennsylvania has the largest number of settlements (55), followed closely by Ohio (54), but Ohio has more church districts. An estimate of the number of Amish living in those two states for 2010, which can be found in an article from the first issue of the Journal of Amish and Plain Anabaptist Studies (Donnermeyer, Anderson, and Cooksey 2012), also estimated their populations to be very close in size.

By number of settlements, New York now ranks third, with 50, but by number of church districts, Indiana is far ahead of the Empire State. The reason is simple: Indiana includes 5 large settlements (all founded in the 1800s). In contrast, Conewango Valley founded in 1949 is the oldest New York settlement, and is one of only two settlements with more than ten church 
districts in the state.

The recent and incredible increase of settlements in New York can be seen in Table 3. Just since January 1, 2010, there are 17 new communities in New York, representing exactly onefourth of the 69 newly established settlements anywhere in Canada and the United States during the second decade of this century. Another 18 communities which still exist today were started in New York from 2000 to 2009. Before this twenty-first century began, New York played host to only 15 settlements that are still active today. Now, it rivals Pennsylvania and Ohio for the number of existing settlements.

Other states have seen similar increases, including Kentucky and Missouri, each with 21 new settlements founded since 2000. Ohio is not far behind, with 20 new settlements, most of which were established in the southeastern or "Appalachian” counties of the Buckeye state. Other notable host states for the number of new Amish communities in the twenty-first century include Wisconsin (16), Michigan (13), Illinois (12), Iowa (12), Minnesota (10), and Pennsylvania (10).

Each decade since 1970 shows a different state where new communities were concentrated. In the 1970s, the leader was Pennsylvania, and in the 1980s, Michigan hosted more new Amish settlements than any other state / province. By the 1990s, it was Wisconsin and Ohio who witnessed the most settlement growth. During the first decade of the twenty-first century, there was a close four-way race, with New York in the lead, followed by Missouri, Ohio, and Kentucky. And, without question, New York continues to be the "go-to" state since this second decade began.

The number of stars on the map of existing Amish communities graphically shows the unprecedented growth of settlements in New York (see Figure 1). The map also shows the spread of Amish settlements into the southern and western regions of the United States and the northern expansion of communities in Maine, Minnesota, and Ontario.

Table 4 accounts for the net growth of Amish settlements, decade by decade, since 1900. The far left column shows each decade. The next column to the right shows the number of settlements which began during each decade which still exist today. The total for all decades of the twentieth century and up to September 30, 2013, is 454. A total of 472 is achieved when the 18 still existing settlements which were founded prior to 1900 are included.

The next column shows the number of settlements founded during each decade which did not survive, followed by the fourth column from the left, which shows the total number of communities started in each decade. Simply, the numbers in the fourth column are a summation of the second and third columns. Altogether, up to September 30, 2013, a total of 625 settlements were started since 1900, with 171 that did not survive. The 454 which remain active represents a survival rate of nearly $73 \%$ and $27 \%$ that failed. 
The two columns on the right side of Table 4 show settlement growth in a different way. The column on the far right displays the net gain in settlements, decade by decade. It is a number created by subtracting the actual number of settlements which became extinct during a decade (second column from the right) from the total founded. All but one decade (1930 to 1939) shows a net increase in settlements. Since the beginning of the 21st century, the net gain is an incredible 178 settlements, which translates to a gain of one settlement every 3.72 weeks, after accounting for settlements which became extinct during this same period.

Table 5 is a list of recently extinct settlements. Notable on this list is Plain City, Ohio, which was over 100 years old before it ceased to function as a community in 2011. Most of the others were settlements that survived less than 20 years before they became extinct, like Salem, Kentucky (1993 to 2011).

Some of the recently extinct communities have already been replaced. These include Coral, Michigan; Kirksville / Gibbs, Missouri; and Ebensburg / Nicktown, Pennsylvania. The names of the replacement settlements in Table 1, however, may be slightly different, depending on the specific addresses and postal codes of the incoming families, as well as what the new Amish there want their community to be called. Of note is that the settlement of Coral is now a second replacement. The original settlement lasted 17 years (2001 to 2008), followed by one short-lived attempt (2008 to 2011).

In keeping with the tradition of previous editions of Amish Settlements across America, we include a number of interesting facts about Amish communities (Table 6). It describes both the rapid growth of settlements and interesting facts about particular states and localities. For example, there are ten settlements which straddle a state border. There are three counties next to each other-Crawford and Mercer Counties in Pennsylvania and Ashtabula County in Ohiowhich contain, either completely or partially, 19 distinct Amish communities. This is likely the largest concentration of settlements anywhere.

The last fact (\#15) presented in Table 6 is a best guess on the number of settlements there will be in both 2020 and 2050. It seems as though each decade is one where it could be said that "growth won't get any higher," and then it does. However, whether the numbers are going up, down or sideways, they are merely hints about the real story. The real story is about family, church, and community, and how the Amish have found room to grow within the North American societies of Canada and the United States. 
Table 1: Settlement Location by State / Province

\begin{tabular}{|c|c|c|}
\hline Settlement Location & $\begin{array}{c}\text { Year } \\
\text { Founded }\end{array}$ & $\begin{array}{l}\text { Church } \\
\text { Districts } \\
\end{array}$ \\
\hline \multicolumn{3}{|l|}{ ARKANSAS } \\
\hline Salem (Fulton County) & 2009 & 1 \\
\hline Rector (Clay County) & 2009 & 1 \\
\hline \multicolumn{3}{|l|}{ COLORADO } \\
\hline Monte Vista (Rio Grande \& Conejos counties) & 2002 & 2 \\
\hline La Jara (Conejos County) & 2004 & 1 \\
\hline Westcliffe (Custer County) & 2008 & 1 \\
\hline Hillside/Cotopaxi (Custer \& Fremont counties) & 2011 & 1 \\
\hline \multicolumn{3}{|l|}{ DELAWARE } \\
\hline Dover/Hartly (Kent County) & 1915 & 10 \\
\hline \multicolumn{3}{|l|}{ FLORIDA } \\
\hline Pinecraft (Sarasota County) & 1927 & 1 \\
\hline \multicolumn{3}{|l|}{ IDAHO } \\
\hline Salmon (Lemhi County) & 2012 & 1 \\
\hline \multicolumn{3}{|l|}{ ILLINOIS } \\
\hline Arthur/Arcola (Douglas, Moultrie \& Cole Counties) & 1864 & 29 \\
\hline Ava (Jackson \& Randolph Counties) & 1991 & 2 \\
\hline Colchester/Macomb (McDonough County) & 1993 & 2 \\
\hline Pleasant Hill/Martinsburg (Pike County) & 1994 & 1 \\
\hline Belle Rive/Opdyke (Jefferson County) & 1995 & 2 \\
\hline Flat Rock (Crawford County) & 1995 & 1 \\
\hline Vienna (Johnson County) & 2000 & 1 \\
\hline Barry (Adams \& Pike Counties) & 2002 & 1 \\
\hline Cisne/Fairfield (Wayne County) & 2002 & 1 \\
\hline Cuba/Lewistown (Fulton County) & 2005 & 1 \\
\hline Carbondale (Jackson County) & 2006 & 1 \\
\hline Oblong (Crawford County) & 2006 & 1 \\
\hline Dale/McLeansboro (Hamilton County) & 2006 & 1 \\
\hline Roseville (Warren County) & 2007 & 1 \\
\hline Harrisburg/Galatia (Saline County) & 2007 & 1 \\
\hline Xenia/Orchardville (Clay \& Wayne Counties) & 2007 & 2 \\
\hline Johnsonville (Wayne County) & 2008 & 1 \\
\hline Sumner/Olney (Richland \& Lawrence Counties) & 2010 & 1 \\
\hline \multicolumn{3}{|l|}{ INDIANA } \\
\hline Elkhart-LaGrange-Noble Counties & 1841 & 153 \\
\hline Nappanee (Elkhart, Kosciusko, Marshall \& St Joseph Counties) & 1841ca & 39 \\
\hline Kokomo/Amboy (Howard \& Miami Counties) & 1848 & 2 \\
\hline $\begin{array}{l}\text { Berne/Monroe/Geneva (Adams, Jay \& Wells Counties, IN \& } \\
\text { Mercer County, OH) }\end{array}$ & 1850 & 54 \\
\hline Grabill/New Haven (Allen County) & 1852 & 19 \\
\hline
\end{tabular}




\begin{tabular}{|c|c|c|}
\hline Settlement Location & \begin{tabular}{|c|} 
Year \\
Founded
\end{tabular} & $\begin{array}{c}\text { Church } \\
\text { Districts }\end{array}$ \\
\hline \multicolumn{3}{|l|}{ INDIANA continued } \\
\hline Montgomery/Odon (Daviess \& Martin Counties) & 1868 & 28 \\
\hline Paoli (Orange County) & 1957 & 1 \\
\hline Hamilton (Steuben County, IN \& Williams County, OH) & 1964 & 1 \\
\hline Milroy (Rush \& Decatur Counties) & 1969 & 3 \\
\hline Salem (Washington County) & 1972 & 1 \\
\hline South Whitley (Whitley County) & 1974 & 2 \\
\hline Salem (Washington County) “Swiss” & 1981 & 3 \\
\hline Vevay (Switzerland \& Jefferson Counties) “Swiss” & 1987 & 3 \\
\hline Rockville (Parke County) & 1991 & 7 \\
\hline Hagerstown/Greensfork (Henry, Randolph \& Wayne Counties) & 1994 & 8 \\
\hline Orleans (Lawrence \& Orange County) & 1994 & 3 \\
\hline Rochester/Akron (Fulton \& Miami Counties) & 1996 & 2 \\
\hline Vevay (Switzerland County) & 2003 & 2 \\
\hline English (Crawford County) & 2007 & 1 \\
\hline Holton/Versailles (Ripley County) & 2008 & 1 \\
\hline Mooreland (Henry and Wayne Counties) & 2008 & 1 \\
\hline Gosport (Owen County) & 2011 & 1 \\
\hline \multicolumn{3}{|l|}{ IOWA } \\
\hline Kalona (Johnson \& Washington Counties) & 1846 & 10 \\
\hline Hazelton/Fairbank (Buchanan County) & 1914 & 7 \\
\hline Milton/Pulaski (Davis \& Van Buren Counties) & 1969 & 3 \\
\hline Bloomfield/Drakesville (Davis County) & 1971 & 9 \\
\hline McIntire/Riceville (Howard \& Mitchell Counties) & 1975 & 2 \\
\hline Edgewood (Clayton \& Delaware Counties) & 1986 & 3 \\
\hline Chariton/Corydon (Lucas County) & 1992 & 1 \\
\hline Redding (Ringgold County) & 1994 & 2 \\
\hline Seymour (Wayne County, IA \& Putnam County, MO) & 1996 & 4 \\
\hline $\begin{array}{l}\text { Lamoni/Davis City (Decatur \& Ringgold Counties, IA \& } \\
\text { Harrison County, MO) }\end{array}$ & 1997 & 3 \\
\hline Waukon (Allamakee County) & 2000 & 1 \\
\hline Diagonal (Ringgold County) & 2002 & 1 \\
\hline Centerville (Appanoose County) & 2002 & 1 \\
\hline Brighton (Jefferson County) & 2003 & 1 \\
\hline Bonaparte (Van Buren County) & 2005 & 2 \\
\hline Murray (Clarke \& Union Counties) & 2006 & 1 \\
\hline Northwood (Worth County) & 2006 & 1 \\
\hline Sigourney (Keokuk County) & 2010 & 1 \\
\hline Clermont (Fayette County) & 2010 & 1 \\
\hline Hopkinton/Delhi (Delaware County) & 2011 & 1 \\
\hline Albia (Monroe County) & 2011 & 1 \\
\hline Williamson/Chariton (Lucas County) & 2011 & 1 \\
\hline
\end{tabular}




\begin{tabular}{|c|c|c|}
\hline Settlement Location & $\begin{array}{c}\text { Year } \\
\text { Founded }\end{array}$ & $\begin{array}{c}\text { Church } \\
\text { Districts }\end{array}$ \\
\hline \multicolumn{3}{|l|}{ KANSAS } \\
\hline Haven/Yoder (Reno County) & 1883 & 3 \\
\hline Hutchinson (Reno County) & 1883 & 2 \\
\hline Garnett (Anderson County) & 1903 & 2 \\
\hline Fort Scott (Bourbon County) & 2005 & 1 \\
\hline Chetopa (Labette County) & 2006 & 2 \\
\hline Parsons (Labette \& Neosho Counties) & 2006 & 2 \\
\hline Marysville/Axtell (Marshall County) & 2007 & 1 \\
\hline \multicolumn{3}{|l|}{ KENTUCKY } \\
\hline Guthrie (Todd County) & 1958 & 3 \\
\hline Crofton (Christian County) & 1972 & 2 \\
\hline Marion (Crittenden County) & 1977 & 4 \\
\hline Dunnville/Columbia (Adair \& Casey Counties) & 1978 & 2 \\
\hline Glasgow (Barren County) & 1982 & 1 \\
\hline Park City/Smith’s Grove (Barren \& Warren Counties) & 1984 & 3 \\
\hline Sonora /Upton (Hardin County) & 1986 & 1 \\
\hline Munfordville/Horse Cave (Grayson \& Hart Counties) & 1989 & 14 \\
\hline Hopkinsville/Pembroke (Christian, Logan \& Trigg Counties) & 1989 & 9 \\
\hline Hardyville/Three Springs (Hart \& Metcalfe Counties) & 1991 & 2 \\
\hline Sonora (Hardin \& Larue Counties) "Swartzentruber” & 1991 & 3 \\
\hline Crab Orchard (Lincoln \& Rockcastle Counties) & 1994 & 4 \\
\hline Hudson/Harned (Breckinridge County) & 1997 & 2 \\
\hline Lewisburg (Logan County) & 1998 & 2 \\
\hline Hillsboro (Fleming County) & 1999 & 2 \\
\hline Princeton (Caldwell \& Lyons Counties) & 2001 & 1 \\
\hline Fancy Farm/Mayfield (Graves \& Hickman Counties) & 2001 & 1 \\
\hline Mayfield (Graves County) "Swartzentruber" & 2002 & 1 \\
\hline Cadiz (Trigg County) & 2002 & 1 \\
\hline Turners Station (Carroll \& Henry Counties) & 2002 & 1 \\
\hline Campbellsville/Mannsville (Taylor County) & 2004 & 1 \\
\hline Flemingsburg/Poplar Grove (Fleming County) "Swiss” & 2004 & 3 \\
\hline Mays Lick (Mason County) & 2004 & 2 \\
\hline Irvington (Breckinridge County) & 2004 & 1 \\
\hline Auburn (Logan County) & 2005 & 1 \\
\hline Tollesboro/Vanceburg (Lewis County) & 2005 & 1 \\
\hline Carlisle (Nicholas County) & 2006 & 1 \\
\hline Owingsville/ "Preston District" (Bath County) "Swiss" & 2006 & 1 \\
\hline Morgantown (Butler County) & 2010 & 1 \\
\hline Willisburg/Springfield (Washington County) & 2010 & 1 \\
\hline Owingsville/Slate Valley (Bath County) “Troyer” & 2011 & 1 \\
\hline Leitchfield (Grayson County) & 2011 & 1 \\
\hline Hawesville (Hancock County) & 2011 & 1 \\
\hline
\end{tabular}




\begin{tabular}{|c|c|c|}
\hline Settlement Location & $\begin{array}{c}\text { Year } \\
\text { Founded }\end{array}$ & $\begin{array}{c}\text { Church } \\
\text { Districts }\end{array}$ \\
\hline \multicolumn{3}{|l|}{ KENTUCKY continued } \\
\hline Gravel Switch (Boyle \& Marion County) & 2011 & 1 \\
\hline Liberty (Casey County) & 2013 & 1 \\
\hline Pleasureville (Henry County) & 2013 & 1 \\
\hline \multicolumn{3}{|l|}{ MAINE } \\
\hline Smyrna Mills/Oakfield (Aroostook County) & 2000 & 1 \\
\hline Fort Fairfield (Aroostook County) & 2007 & 1 \\
\hline Unity (Waldo County) & 2008 & 1 \\
\hline Sherman (Aroostook County) & 2011 & 1 \\
\hline Hodgdon (Aroostook County) & 2012 & 1 \\
\hline \multicolumn{3}{|l|}{ MARYLAND } \\
\hline Oakland (Garrett County) & 1850 & 1 \\
\hline Mechanicsville (St. Mary’s County) & 1940 & 9 \\
\hline Cecilton (Cecil County) & 1999 & 1 \\
\hline \multicolumn{3}{|l|}{ MICHIGAN } \\
\hline Centreville (St. Joseph County) & 1910 & 11 \\
\hline Camden (Hillsdale County) & 1956 & 4 \\
\hline California Township (Branch County) & 1960 & 6 \\
\hline Mio (Oscoda County) & 1970 & 3 \\
\hline Bronson (Branch County, MI) & 1971 & 1 \\
\hline Greenville/Six Lakes (Montcalm County) & 1973 & 4 \\
\hline Quincy/Coldwater (Branch \& Hillsdale Counties) & 1977 & 4 \\
\hline Charlotte/Vermontville (Eaton County) & 1977 & 3 \\
\hline Gladwin/Beaverton (Clare \& Gladwin County) “Swartzentruber” & 1979 & 3 \\
\hline Reading/Hillsdale (Branch County) & 1979 & 2 \\
\hline Gladwin (Gladwin County) & 1980 & 3 \\
\hline Clare (Clare \& Isabella Counties) & 1981 & 4 \\
\hline Rosebush (Isabella County) & 1981 & 1 \\
\hline Stanwood/Morley (Mecosta County) & 1982 & 6 \\
\hline Blanchard (Isabella County) & 1983 & 2 \\
\hline Marlette/Brown City (Sanilac County) & 1987 & 3 \\
\hline Homer (Calhoun County) & 1989 & 2 \\
\hline Evart/Sears (Osceola County) & 1989 & 1 \\
\hline Fremont/Holton (Newaygo, Muskegon \& Oceana Counties) & 1990 & 2 \\
\hline Cass City/Ubly (Huron, Sanilac \& Tuscola Counties) & 1993 & 4 \\
\hline Manton (Wexford County) & 1993 & 2 \\
\hline Newaygo (Newaygo County) & 1994 & 2 \\
\hline Marion (Osceola County) & 1995 & 1 \\
\hline Ossineke (Alpena County) & 1995 & 1 \\
\hline Osseo/Pittsford (Hillsdale County) & 1997 & 2 \\
\hline Bloomingdale (Van Buren County) & 1998 & 1 \\
\hline McBain (Missaukee County) & 2000 & 2 \\
\hline
\end{tabular}




\begin{tabular}{|c|c|c|}
\hline Settlement Location & $\begin{array}{c}\text { Year } \\
\text { Founded }\end{array}$ & $\begin{array}{l}\text { Church } \\
\text { Districts }\end{array}$ \\
\hline \multicolumn{3}{|l|}{ MICHIGAN continued } \\
\hline LeRoy (Osceola County) & 2000 & 1 \\
\hline Hersey (Osceola County) & 2003 & 1 \\
\hline Coldwater/Kinderhook (Branch County) & 2004 & 1 \\
\hline Hastings (Barry County) & 2006 & 1 \\
\hline Hale/Whittemore (Iosco County) & 2006 & 1 \\
\hline Vestaburg (Gratiot \& Montcalm Counties) & 2007 & 1 \\
\hline Engadine (Mackinac County) & 2008 & 1 \\
\hline North Adams (Hillsdale County) & 2010 & 1 \\
\hline Coral (Montcalm County) & 2011 & 1 \\
\hline Hawks (Presque Isle County) & 2012 & 1 \\
\hline Tustin (Osceola County) & 2012 & 1 \\
\hline Reed City/Chase (Lake \& Osceola Counties) & 2012 & 1 \\
\hline \multicolumn{3}{|l|}{ MINNESOTA } \\
\hline Wadena (Otter Tail \& Wadena Counties) & 1972 & 2 \\
\hline Bertha/Hewitt (Todd County) & 1973 & 1 \\
\hline Canton /Harmony (Fillmore County) & 1974 & 6 \\
\hline Utica/St. Charles (Winona County) & 1975 & 3 \\
\hline Granger/Cresco (Fillmore County, MN \& Howard County, IA) & 1993 & 3 \\
\hline Long Prairie/Osakis (Todd County) & 1995 & 2 \\
\hline Clearbrook/Gonvick (Clearwater County) & 2000 & 1 \\
\hline Clarissa/Browerville (Todd County) & 2001 & 2 \\
\hline Bertha/Staples (Todd County) "Swartzentruber” & 2003 & 1 \\
\hline Frazee/Wolf Lake (Becker County) & 2007 & 2 \\
\hline Fosston (Polk County) & 2007 & 1 \\
\hline Fertile (Norman \& Polk Counties) & 2007 & 1 \\
\hline Eagle Bend (Todd County) & 2007 & 1 \\
\hline Mora (Kanabec County) & 2011 & 1 \\
\hline Lengby (Polk County) & 2012 & 1 \\
\hline Milaca (Mille Lacs County) & 2012 & 1 \\
\hline \multicolumn{3}{|l|}{ MISSISSIPPI } \\
\hline Randolph (Pontotoc County) & 1995 & 1 \\
\hline \multicolumn{3}{|l|}{ MISSOURI } \\
\hline Bowling Green/Curryville (Pike County) & 1947 & 3 \\
\hline Jamesport (Daviess County) & 1953 & 8 \\
\hline Clark/Madison (Audrain, Monroe \& Randolph Counties) & 1954 & 10 \\
\hline Anabel (Macon County) & 1957 & 1 \\
\hline Seymour/Fordland (Webster County) & 1968 & 15 \\
\hline Windsor (Benton, Henry County, Johnson \& Pettis Counties) & 1975 & 3 \\
\hline LaPlata (Adair \& Macon Counties) & 1976 & 4 \\
\hline Dixon (Pulaski County) & 1980 & 1 \\
\hline Kahoka (Clark County) & 1985 & 1 \\
\hline Canton (Lewis County) & 1986 & 3 \\
\hline
\end{tabular}




\begin{tabular}{|c|c|c|}
\hline Settlement Location & $\begin{array}{c}\text { Year } \\
\text { Founded }\end{array}$ & $\begin{array}{c}\text { Church } \\
\text { Districts }\end{array}$ \\
\hline \multicolumn{3}{|l|}{ MISSOURI continued } \\
\hline Humansville/Dunnegan (Polk County) & 1987 & 1 \\
\hline Carrollton/Bogard (Carroll County) & 1990 & 2 \\
\hline Verona (Lawrence County) & 1990 & 2 \\
\hline Mt. Vernon (Lawrence County) & 1995 & 1 \\
\hline Spickard/Princeton (Grundy County) "Swartzentruber” & 1997 & 3 \\
\hline Mountain Grove (Wright County) & 1998 & 1 \\
\hline Bethany (Harrison County) & 1999 & 1 \\
\hline Stanberry/Ravenwood (Gentry County) & 2000 & 3 \\
\hline Unionville (Putnam County) & 2000 & 1 \\
\hline Harwood/Nevada (Vernon County) & 2000 & 1 \\
\hline Lamar (Barton County) & 2002 & 2 \\
\hline Osceola (St. Clair County) & 2002 & 2 \\
\hline Prairie Home/Boonville (Cooper County) & 2003 & 2 \\
\hline Greentop/Queen City (Schuyler County) & 2003 & 2 \\
\hline El Dorado Springs (Cedar County) & 2004 & 1 \\
\hline Mercer (Mercer County, MO \& Decatur County, IA) & 2005 & 1 \\
\hline Hartshorn/Summersville (Shannon County) & 2006 & 1 \\
\hline Keytesville (Chariton County) & 2007 & 1 \\
\hline Bolivar/Flemington (Hickory \& Polk Counties) & 2007 & 1 \\
\hline Princeton/Modena (Mercer County) & 2007 & 2 \\
\hline Monroe City (Monroe County) & 2008 & 1 \\
\hline Downing (Schuyler County) & 2008 & 1 \\
\hline Ethel/New Cambria (Macon County) & 2009 & 1 \\
\hline Licking (Texas County) & 2009 & 1 \\
\hline Kirksville/Gibbs (Adair County) & 2010 & 1 \\
\hline El Dorado Springs (Cedar County) "Swartzentruber" & 2010 & 1 \\
\hline Hamilton (Caldwell County) & 2011 & 1 \\
\hline Rocky Comfort (McDonald County) & 2012 & 1 \\
\hline \multicolumn{3}{|l|}{ MONTANA } \\
\hline Rexford (Lincoln County) & 1974 & 1 \\
\hline St. Ignatius (Lake County) & 1997 & 2 \\
\hline Ashland/Forsyth (Rosebud County) & 1997 & 1 \\
\hline Moore/Lewistown (Fergus County) & 2008 & 1 \\
\hline \multicolumn{3}{|l|}{ NEBRASKA } \\
\hline Verdigre (Knox County) & 2003 & 1 \\
\hline Ewing/Orchard (Holt County) & 2005 & 1 \\
\hline Pawnee City (Pawnee County) & 2007 & 1 \\
\hline Verdigre (Knox County) “Swartzentruber” & 2010 & 1 \\
\hline \multicolumn{3}{|l|}{ NEW YORK } \\
\hline Conewango Valley (Cattaraugus County) & 1949 & 15 \\
\hline Norfolk/Norwood (St. Lawrence County) & 1974 & 1 \\
\hline Heuvelton (St. Lawrence County) & 1975 & 12 \\
\hline
\end{tabular}




\begin{tabular}{|c|c|c|}
\hline Settlement Location & $\begin{array}{c}\text { Year } \\
\text { Founded }\end{array}$ & $\begin{array}{l}\text { Church } \\
\text { Districts }\end{array}$ \\
\hline \multicolumn{3}{|l|}{ NEW YORK continued } \\
\hline Dewittville/Mayville (Chautauqua County) & 1976 & 2 \\
\hline Cylmer/Panama (Chautauqua County) & 1976 & 8 \\
\hline Prattsburgh (Steuben County) & 1979 & 1 \\
\hline Romulus/Ovid (Seneca County) & 1981 & 4 \\
\hline Friendship/Belfast (Allegany County) & 1982 & 2 \\
\hline Woodhull/Jasper (Steuben County) & 1983 & 7 \\
\hline Fort Plain/Fonda (Montgomery County) & 1986 & 5 \\
\hline Fillmore (Allegany \& Wyoming Counties) & 1988 & 4 \\
\hline Addison (Steuben County) & 1990 & 2 \\
\hline Clyde (Seneca \& Wayne Counties) & 1997 & 3 \\
\hline Lyndonville/Medina (Orleans County) & 1998 & 2 \\
\hline Lowville (Lewis County) & 1999 & 2 \\
\hline Richfield Springs (Herkimer \& Otsego Counties) & 2000 & 2 \\
\hline Malone/Burke (Franklin County) & 2002 & 1 \\
\hline South Columbia Township (Herkimer County) & 2002 & 1 \\
\hline Summer Hill (Cayuga County) & 2003 & 1 \\
\hline Little Falls (Herkimer County) & 2004 & 1 \\
\hline Nicholville/Potsdam (Franklin \& St. Lawrence Counties) & 2004 & 2 \\
\hline Fultonville/Glen (Montgomery County) & 2005 & 2 \\
\hline LaFargeville (Jefferson County) & 2005 & 2 \\
\hline Williamstown/Pulaski (Oswego County) & 2006 & 2 \\
\hline Georgetown/Eaton (Madison County) & 2006 & 1 \\
\hline Mohawk Valley/Fort Plain (Montgomery County) & 2006 & 3 \\
\hline Little Valley/East Otto (Cattaraugus County) & 2006 & 1 \\
\hline Canastota (Madison County) & 2006 & 1 \\
\hline Poland (Herkimer \& Oneida Counties) “Swartzentruber” & 2007 & 1 \\
\hline Lyons (Wayne County) & 2009 & 1 \\
\hline Bombay (Franklin County) & 2009 & 1 \\
\hline Angelica (Allegany County) & 2009 & 2 \\
\hline Franklinville (Cattaraugus County) & 2009 & 1 \\
\hline Camden/Westdale (Oneida County) & 2010 & 1 \\
\hline North Rose (Wayne County) & 2010 & 1 \\
\hline Oriskany Falls/Augusta (Madison \& Oneida Counties) & 2010 & 1 \\
\hline Marathon (Cortland County) & 2010 & 2 \\
\hline Mount Morris (Livingston County) & 2010 & 1 \\
\hline New Berlin (Chenango \& Otsego Counties) & 2010 & 2 \\
\hline Springwater/Dansville (Livingston County) & 2010 & 1 \\
\hline Mayville (Chautauqua County) & 2011 & 1 \\
\hline Ellenburg Center (Clinton County) & 2011 & 1 \\
\hline Remsen/Rome/Holland Patent (Oneida County) & 2011 & 1 \\
\hline Delevan (Cattaraugus County) “Swartzentruber” & 2011 & 1 \\
\hline
\end{tabular}




\begin{tabular}{|c|c|c|}
\hline Settlement Location & $\begin{array}{c}\text { Year } \\
\text { Founded }\end{array}$ & $\begin{array}{c}\text { Church } \\
\text { Districts }\end{array}$ \\
\hline \multicolumn{3}{|l|}{ NEW YORK continued } \\
\hline Frewsburg (Chautauqua County) & 2011 & 1 \\
\hline Philadelphia (Jefferson County) & 2012 & 1 \\
\hline Lee Center/Ava (Oneida County) & 2012 & 1 \\
\hline Pulteney/Hammondsport (Steuben County) & 2012 & 1 \\
\hline Wellsville (Alleghany County) & 2013 & 1 \\
\hline Lyons (Wayne County) “Troyer” & 2013 & 1 \\
\hline \multicolumn{3}{|l|}{ NORTH CAROLINA } \\
\hline Union Grove (Iredell \& Yadkin Counties) & 1985 & 1 \\
\hline \multicolumn{3}{|l|}{ OHIO } \\
\hline $\begin{array}{l}\text { Greater Holmes (Coshocton, Holmes, Stark, Tuscarawas, \& } \\
\text { Wayne Counties) }\end{array}$ & 1808 & 238 \\
\hline Greater Geauga (Ashtabula, Geauga, Portage \& Trumbull Counties) & 1886 & 105 \\
\hline Hicksville (Defiance County) & 1914 & 1 \\
\hline Lodi/Homerville (Ashland, Medina \& Wayne Counties) & 1952 & 14 \\
\hline Kenton/Mt. Victory (Hardin \& Marion Counties) & 1953 & 8 \\
\hline Ashland/Shiloh (Ashland, Huron \& Richland Counties) & 1954 & 6 \\
\hline Lakeville/Big Prairie (Holmes County) & 1962 & 3 \\
\hline Danville/Butler (Knox \& Richland Counties) & 1964 & 5 \\
\hline $\begin{array}{l}\text { Peoli/Port Washington (Guernsey \& Tuscarawas Counties) } \\
\text { "Swartzentruber” }\end{array}$ & 1969 & 3 \\
\hline Fredericktown/Bellville (Knox, Morrow \& Richland Counties) & 1972 & 11 \\
\hline Belle Center (Logan County) & 1974 & 3 \\
\hline Kinsman (Trumbull County, OH \& Mercer County, PA) & 1975 & 1 \\
\hline West Union (Adams County) & 1976 & 4 \\
\hline Chesterhill/Stockport (Morgan County) & 1978 & 1 \\
\hline Carrollton (Carroll County) & 1982 & 4 \\
\hline Lewisville (Monroe County) & 1987 & 6 \\
\hline Utica/Gambier (Knox \& Licking Counties) & 1987 & 4 \\
\hline Laurelville/Kingston (Hocking \& Pickaway Counties) & 1988 & 1 \\
\hline Bremen (Fairfield County) & 1989 & 1 \\
\hline Brinkhaven/Danville (Holmes \& Knox Counties) & 1990 & 4 \\
\hline Walhonding/Warsaw (Coshocton \& Knox Counties) & 1990 & 1 \\
\hline Loudonville/McKay (Ashland County) & 1991 & 1 \\
\hline Dorset/Cherry Valley (Ashtabula County) & 1991 & 2 \\
\hline Salesville/Quaker City (Guernsey County) & 1991 & 2 \\
\hline Andover (Ashtabula County) & 1992 & 2 \\
\hline Barnesville (Belmont \& Monroe Counties) & 1993 & 2 \\
\hline Gallipolis (Gallia County) & 1993 & 5 \\
\hline Glenmont/Brinkhaven (Coshocton \& Holmes County) & 1994 & 2 \\
\hline Pierpont/Conneaut (Ashtabula County, OH \& Crawford County, PA) & 1994 & 5 \\
\hline De Graff (Logan County) & 1994 & 3 \\
\hline Beaver (Pike County) & 1994 & 2 \\
\hline
\end{tabular}




\begin{tabular}{|c|c|c|}
\hline Settlement Location & $\begin{array}{c}\text { Year } \\
\text { Founded }\end{array}$ & $\begin{array}{l}\text { Church } \\
\text { Districts }\end{array}$ \\
\hline \multicolumn{3}{|l|}{ OHIO continued } \\
\hline Adamsville (Muskingum County) & 1997 & 2 \\
\hline Williamsfield (Ashtabula County) & 1997 & 1 \\
\hline Middlebourne (Guernsey County) & 1998 & 2 \\
\hline Howard/”East Knox” (Knox County) & 2000 & 3 \\
\hline Oak Hill (Jackson County) & 2001 & 2 \\
\hline Scio (Harrison County) & 2001 & 2 \\
\hline Piedmont (Belmont \& Harrison County) & 2001 & 1 \\
\hline McArthur (Vinton County) & 2003 & 1 \\
\hline Vinton (Gallia and Vinton Counties) & 2004 & 1 \\
\hline Kilgore (Carroll County) & 2005 & 2 \\
\hline Hillsboro/Leesburg (Highland County) & 2006 & 2 \\
\hline Somerset/Glenford (Perry County) & 2006 & 1 \\
\hline Peeples/Hillsboro (Adams \& Highland Counties) & 2006 & 1 \\
\hline Pomeroy (Meigs County) & 2006 & 1 \\
\hline Freeport (Harrison County) & 2006 & 1 \\
\hline Newcomerstown (Tuscarawas County) & 2007 & 1 \\
\hline Londonderry (Ross \& Vinton Counties) & 2007 & 1 \\
\hline Frazeysburg (Muskingum County) & 2007 & 1 \\
\hline Pleasant City (Guernsey \& Noble Counties) & 2007 & 1 \\
\hline Cherry Valley (Ashtabula County) & 2010 & 1 \\
\hline Marshall/Hillsboro (Highland County) & 2010 & 1 \\
\hline Rogers (Columbiana County) & 2010 & 1 \\
\hline Jefferson (Ashtabula County) & 2011 & 1 \\
\hline \multicolumn{3}{|l|}{ OKLAHOMA } \\
\hline Chouteau/Inola (Mayes \& Rogers Counties) & 1910 & 4 \\
\hline Clarita/Coalgate (Coal County) & 1978 & 1 \\
\hline Welch (Craig County) & 2010 & 1 \\
\hline Westville (Adair County) & 2012 & 1 \\
\hline \multicolumn{3}{|l|}{ ONTARIO } \\
\hline Milverton/Millbank (Perth \& Waterloo Counties) & 1824 & 9 \\
\hline Aylmer (Elgin County) & 1953 & 3 \\
\hline Norwich (Oxford County) & 1954 & 4 \\
\hline Chesley (Grey County) “Swartzentruber” & 1954 & 3 \\
\hline Lakeside/St. Mary’s (Oxford County) & 1958 & 1 \\
\hline Mossley (Middlesex County) & 1962 & 2 \\
\hline Lucknow (Bruce \& Huron Counties) & 1973 & 5 \\
\hline Chesley (Bruce \& Grey Counties) & 1979 & 1 \\
\hline Kincardine/Tiverton (Bruce County) & 1995 & 2 \\
\hline Clifford/Harriston (Perth \& Wellington Counties) & 1998 & 1 \\
\hline Cameron/Lindsay (Victoria County) & 1998 & 1 \\
\hline Powassan (Parry Sound County) & 2001 & 1 \\
\hline Stirling/Belleville (Hastings County) & 2005 & 1 \\
\hline
\end{tabular}




\begin{tabular}{|c|c|c|}
\hline Settlement Location & $\begin{array}{c}\text { Year } \\
\text { Founded }\end{array}$ & $\begin{array}{c}\text { Church } \\
\text { Districts }\end{array}$ \\
\hline \multicolumn{3}{|l|}{ ONTARIO continued } \\
\hline Iron Bridge (Algoma County) & 2008 & 1 \\
\hline Englehart/New Liskeard (Timiskaming District) & 2009 & 1 \\
\hline Earlton (Timiskaming District) & 2013 & 1 \\
\hline \multicolumn{3}{|l|}{ PENNSYLVANIA } \\
\hline Lancaster/Chester Counties & са. 1760 & 187 \\
\hline Meyersdale/Springs (Somerset County, PA \& Garrett County, MD) & ca. 1772 & 5 \\
\hline Belleville/Reedsville (Huntingdon \& Mifflin Counties) & 1791 & 26 \\
\hline New Wilmington (Lawrence \& Mercer Counties) & 1847 & 19 \\
\hline Enon Valley (Beaver \& Lawrence Counties) & 1924 & 1 \\
\hline Atlantic (Crawford \& Mercer Counties) & 1924 & 7 \\
\hline Myerstown (Berks \& Lebanon Counties) & 1941 & 7 \\
\hline Mercer/Soneboro (Mercer County) & 1942 & 5 \\
\hline Mifflintown/Port Royal (Juniata County) & 1950 & 9 \\
\hline Aaronsburg/Woodward (Centre County) & 1950 & 3 \\
\hline Winfield (Snyder \& Union Counties) & 1959 & 1 \\
\hline Smicksburg (Armstrong \& Indiana Counties) & 1962 & 20 \\
\hline McClure (Snyder County) & 1965 & 3 \\
\hline Spartansburg (Crawford, Erie \& Warren Counties) & 1966 & 10 \\
\hline LeRaysville/Rome (Bradford County) & 1966 & 1 \\
\hline Rebersburg/“Brush Valley” (Centre County) & 1967 & 6 \\
\hline Dry Run/“Path Valley” (Franklin County) & 1968 & 5 \\
\hline Conneautville (Crawford County) & 1969 & 2 \\
\hline Sugar Grove (Warren County, PA \& Chautauqua County, NY) & 1969 & 8 \\
\hline Turbotville/Danville (Montour \& Northumberland Counties) & 1970 & 3 \\
\hline Newburg/Cumberland Valley (Cumberland \& Franklin Counties) & 1971 & 6 \\
\hline Guys Mills (Crawford County) & 1972 & 3 \\
\hline Troutville/Punxsutawney (Clearfield \& Jefferson Counties) & 1972 & 12 \\
\hline Loganton/“Sugar Valley” (Clinton County) & 1972 & 7 \\
\hline Tionesta/Fryburg (Forest County) & 1972 & 2 \\
\hline Howard/Mill Hall “Nittany Valley” (Centre \& Clinton Counties) & 1973 & 6 \\
\hline $\begin{array}{l}\text { Bloomsburg/Danville (Columbia, Montour \& Northumberland } \\
\text { Counties) }\end{array}$ & 1974 & 4 \\
\hline Delta (York County) & 1975 & 3 \\
\hline Loysville/Blain (Perry County) & 1975 & 5 \\
\hline $\begin{array}{l}\text { Allenwood/"White Deer Valley" (Lycoming \& } \\
\text { Northumberland Counties) }\end{array}$ & 1976 & 4 \\
\hline Millersburg/“Lykens Valley” (Dauphin \& Schuykill Counties) & 1978 & 8 \\
\hline Spring Mills/“Penns Valley” (Centre County) & 1979 & 3 \\
\hline Pocahontas (Somerset County) & 1980 & 1 \\
\hline Union City (Crawford \& Erie Counties) & 1983 & 2 \\
\hline Clintonville/Harrisville (Venango County) & 1983 & 3 \\
\hline Williamsport/”Nippenose Valley” (Clinton \& Lycoming Counties) & 1985 & 2 \\
\hline
\end{tabular}




\begin{tabular}{|c|c|c|}
\hline Settlement Location & \begin{tabular}{|c|} 
Year \\
Founded
\end{tabular} & $\begin{array}{c}\text { Church } \\
\text { Districts }\end{array}$ \\
\hline \multicolumn{3}{|l|}{ PENNSYLVANIA continued } \\
\hline Linesville (Crawford County) & 1985 & 2 \\
\hline Tyrone/Sinking Valley (Blair County) & 1988 & 2 \\
\hline Fredonia/Greenville (Mercer County) & 1990 & 2 \\
\hline Berlin/Shanksville (Somerset County) & 1992 & 1 \\
\hline Dornsife (Northumberland County) & 1993 & 2 \\
\hline Paxinos/ "Irish Valley” (Northumberland County) & 1997 & 1 \\
\hline Homer City (Indiana County) & 1997 & 1 \\
\hline Uylsses (Potter County) & 1998 & 2 \\
\hline Hazen/Brookville (Jefferson County) & 1999 & 3 \\
\hline Emlenton/Sligo (Clarion County) & 2000 & 2 \\
\hline Knox (Clarion County) & 2000 & 3 \\
\hline Canton (Bradford County) & 2001 & 1 \\
\hline Ringgold/Mayport (Armstrong \& Jefferson Counties) & 2002 & 1 \\
\hline Johnsonburg/Rossiter (Jefferson County) & 2005 & 3 \\
\hline Saegertown/Cambridge Springs (Crawford County) & 2006 & 1 \\
\hline Greenville (Mercer County) & 2006 & 1 \\
\hline Carlton (Mercer County) & 2010 & 1 \\
\hline Nicktown/North Cambria (Cambria County) & 2013 & 1 \\
\hline Westover (Clearfield County) & 2013 & 1 \\
\hline \multicolumn{3}{|l|}{ SOUTH DAKOTA } \\
\hline Tripp (Hutchinson County) & 2010 & 1 \\
\hline \multicolumn{3}{|l|}{ TENNESSEE } \\
\hline Ethridge (Lawrence County) & 1944 & 10 \\
\hline Huntingdon/Bruceton (Carroll) & 1975 & 1 \\
\hline McKenzie (Carroll \& Weakley Counties) & 1998 & 1 \\
\hline Summertown (Lewis County) & 2000 & 1 \\
\hline Adamsville (McNairy County) & 2009 & 1 \\
\hline Bradford (Gibson County) & 2009 & 1 \\
\hline Deer Lodge (Morgan County) & 2013 & 1 \\
\hline \multicolumn{3}{|l|}{ TEXAS } \\
\hline Beeville (Bee County) & 1999 & 1 \\
\hline \multicolumn{3}{|l|}{ VIRGINIA } \\
\hline Pearisburg (Giles County) & 1993 & 1 \\
\hline Charlotte Court House (Charlotte County) & 1997 & 2 \\
\hline Nathalie (Halifax County) & 2005 & 2 \\
\hline Rose Hill/Jonesville (Lee County) & 2008 & 1 \\
\hline Tazewell/Burkes Garden (Tazewell County) & 2012 & 1 \\
\hline Chatham (Pittsylvania County) & 2013 & 1 \\
\hline \multicolumn{3}{|l|}{ WEST VIRGINIA } \\
\hline Letart (Mason County) & 1996 & 1 \\
\hline Palestine (Wirt County) & 2004 & 1 \\
\hline Forest Hill/Hinton (Summers County) & 2006 & 1 \\
\hline
\end{tabular}




\begin{tabular}{|c|c|c|}
\hline Settlement Location & \begin{tabular}{|c|} 
Year \\
Founded
\end{tabular} & $\begin{array}{c}\text { Church } \\
\text { Districts }\end{array}$ \\
\hline \multicolumn{3}{|l|}{ WISCONSIN } \\
\hline Medford (Taylor County) & 1920 & 3 \\
\hline Blair (Trempealeau County) & 1960 & 3 \\
\hline Cashton (Monroe \& Vernon Counties) & 1966 & 13 \\
\hline Wilton/Tomah (Monroe County) & 1969 & 8 \\
\hline Spencer (Clark \& Marathon Counties) & 1970 & 1 \\
\hline Chetek (Barron County) & 1974 & 1 \\
\hline Greenwood/Willard (Clark County) & 1975 & 3 \\
\hline Evansville/Brodhead (Green \& Rock Counties) & 1975 & 1 \\
\hline Kingston/Dalton (Columbia, Green Lake \& Marquette Counties) & 1977 & 12 \\
\hline Augusta/Fairfield (Eau Claire County) & 1978 & 8 \\
\hline Granton (Clark County) & 1981 & 6 \\
\hline Wautoma/Coloma (Marquette/Waushara Counties) & 1983 & 4 \\
\hline Hillsboro (Richland \& Vernon Counties) & 1985 & 8 \\
\hline Bonduel (Shawano County) & 1987 & 2 \\
\hline LaValle (Sauk County) & 1988 & 3 \\
\hline Loganville (Sauk County) & 1988 & 2 \\
\hline Loyal (Clark County) “Swartzentruber” & 1989 & 5 \\
\hline Readstown (Crawford, Richland \& Vernon Counties) & 1990 & 3 \\
\hline Athens (Marathon \& Taylor Counties) & 1990 & 2 \\
\hline Mondovi (Buffalo \& Pepin Counties) & 1991 & 2 \\
\hline Owen/Unity (Clark County) & 1991 & 1 \\
\hline New Holstein/Elkhart Lake (Calumet \& Fond du Lac Counties) & 1992 & 1 \\
\hline Viroqua/Dach Ridge (Vernon County) & 1992 & 1 \\
\hline Beetown/Bloomington (Grant County) & 1993 & 1 \\
\hline Chaseburg/Viroqua (Vernon County) & 1994 & 3 \\
\hline Lookout/Wulff Valley (Buffalo County) & 1995 & 1 \\
\hline Marion (Waupaca County) & 1995 & 2 \\
\hline Livingston (Grant County) & 1997 & 1 \\
\hline New Auburn (Chippewa County) & 1997 & 2 \\
\hline Fennimore (Grant County) & 1998 & 3 \\
\hline Taylor (Jackson \& Trempealeau Counties) & 1998 & 2 \\
\hline Edgar/Stratford (Marathon County) & 1999 & 1 \\
\hline Platteville/Darlington (Grant \& Lafayette Counties) & 1999 & 5 \\
\hline Milladore (Portage \& Wood Counties) & 2000 & 1 \\
\hline Reedsville (Manitowoc County) & 2002 & 1 \\
\hline Ettrick/Franklin (Jackson County) & 2002 & 1 \\
\hline Cornell (Chippewa County) & 2002 & 1 \\
\hline Sheldon (Rusk \& Taylor Counties) & 2002 & 1 \\
\hline Arpin/Vesper (Wood County) & 2002 & 2 \\
\hline Bangor (LaCrosse \& Monroe Counties) & 2003 & 1 \\
\hline Black River Falls (Jackson County) & 2004 & 1 \\
\hline Neillsville (Clark County) & 2005 & 1 \\
\hline
\end{tabular}




\begin{tabular}{|c|c|c|}
\hline \multicolumn{1}{|c|}{ Settlement Location } & $\begin{array}{c}\text { Year } \\
\text { Founded }\end{array}$ & $\begin{array}{c}\text { Church } \\
\text { Districts }\end{array}$ \\
\hline WISCONSIN continued & 2007 & 1 \\
\hline Gilman (Taylor County) & 2008 & 1 \\
\hline Lublin (Taylor County) & 2011 & 1 \\
\hline Prairie Farms/Clear Lake (Barron and Polk Counties) & 2012 & 1 \\
\hline Frederic (Polk County) & 2012 & 1 \\
\hline Woodville (St. Croix County) & 2013 & 1 \\
\hline Luck (Polk County) & 2013 & 1 \\
\hline Monroe (Green County) & & \\
\hline WYOMING & 2011 & 1 \\
\hline Hulett (Crook County)
\end{tabular}

Table 2: Amish Settlements and Church Districts by State / Province

\begin{tabular}{lcc} 
State & Settlements & Districts \\
\hline Arkansas & 2 & 2 \\
Colorado & 4 & 5 \\
Delaware & 1 & 10 \\
Florida & 1 & 1 \\
Idaho & 1 & 1 \\
Illinois & 18 & 50 \\
Indiana & 22 & 335 \\
Iowa & 22 & 57 \\
Kansas & 7 & 13 \\
Kentucky & 36 & 78 \\
Maine & 5 & 5 \\
Maryland & 3 & 11 \\
Michigan & 39 & 92 \\
Minnesota & 16 & 29 \\
Mississippi & 1 & 1 \\
Missouri & 38 & 87 \\
Montana & 4 & 5 \\
Nebraska & 4 & 4 \\
New York & 50 & 115 \\
North Carolina & 1 & 1 \\
Ohio & 54 & 475 \\
Oklahoma & 4 & 7 \\
Ontario & 16 & 37 \\
Pennsylvania & 55 & 430 \\
South Dakota & 1 & 1 \\
Tennessee & 7 & 16 \\
Texas & 1 & 1 \\
Virginia & 6 & 8 \\
West Virginia & 3 & 3 \\
Wisconsin & 49 & 131 \\
Wyoming & 1 & 1 \\
\hline Total & 472 & 2,012
\end{tabular}


Table 3: Chronology of Existing Settlements by State / Province

\begin{tabular}{|c|c|c|c|c|c|c|c|c|c|c|c|c|c|c|}
\hline State & $\begin{array}{c}\text { Before } \\
1900\end{array}$ & $\begin{array}{c}1900- \\
1909\end{array}$ & $\begin{array}{c}1910- \\
1919\end{array}$ & $\begin{array}{c}1920- \\
1929\end{array}$ & $\begin{array}{r}1930- \\
1939\end{array}$ & $\begin{array}{c}1940- \\
1949\end{array}$ & $\begin{array}{c}1950- \\
1959\end{array}$ & $\begin{array}{c}1960- \\
1969\end{array}$ & $\begin{array}{c}1970- \\
1979\end{array}$ & $\begin{array}{c}1980- \\
1989\end{array}$ & $\begin{array}{c}1990- \\
1999\end{array}$ & $\begin{array}{c}2000- \\
2009\end{array}$ & $\begin{array}{l}2010- \\
2013\end{array}$ & Total \\
\hline \multirow{31}{*}{$\begin{array}{l}\text { Arkansas } \\
\text { Colorado } \\
\text { Delaware } \\
\text { Florida } \\
\text { Idaho } \\
\text { Illinois } \\
\text { Indiana } \\
\text { Iowa } \\
\text { Kansas } \\
\text { Kentucky } \\
\text { Maine } \\
\text { Maryland } \\
\text { Michigan } \\
\text { Minnesota } \\
\text { Mississippi } \\
\text { Missouri } \\
\text { Montana } \\
\text { Nebraska } \\
\text { New York } \\
\text { North Carolina } \\
\text { Ohio } \\
\text { Oklahoma } \\
\text { Ontario } \\
\text { Pennsylvania } \\
\text { South Dakota } \\
\text { Tennessee } \\
\text { Texas } \\
\text { Virginia } \\
\text { West Virginia } \\
\text { Wisconsin } \\
\text { Wyoming } \\
\end{array}$} & & & & & & & & & & & & 2 & & 2 \\
\hline & & & & & & & & & & & & 3 & 1 & 4 \\
\hline & & & 1 & & & & & & & & & & & 1 \\
\hline & & & & 1 & & & & & & & & & & 1 \\
\hline & & & & & & & & & & & & & 1 & 1 \\
\hline & 1 & & & & & & & & & & 5 & 11 & 1 & 18 \\
\hline & 6 & & & & & & 1 & 2 & 2 & 2 & 4 & 4 & 1 & 22 \\
\hline & 1 & & 1 & & & & & 1 & 2 & 1 & 4 & 7 & 5 & 22 \\
\hline & 2 & 1 & & & & & & & & & & 4 & & 7 \\
\hline & & & & & & & 1 & & 3 & 5 & 6 & 13 & 8 & 36 \\
\hline & & & & & & & & & & & & 3 & 2 & 5 \\
\hline & 1 & & & & & 1 & & & & & 1 & & & 3 \\
\hline & & & 1 & & & & 1 & 1 & 7 & 8 & 8 & 8 & 5 & 39 \\
\hline & & & & & & & & & 4 & & 2 & 7 & 3 & 16 \\
\hline & & & & & & & & & & & 1 & & & 1 \\
\hline & & & & & & 1 & 3 & 1 & 2 & 4 & 6 & 17 & 4 & 38 \\
\hline & & & & & & & & & 1 & & 2 & 1 & & 4 \\
\hline & & & & & & & & & & & & 3 & 1 & 4 \\
\hline & & & & & & 1 & & & 5 & 5 & 4 & 18 & 17 & 50 \\
\hline & & & & & & & & & & 1 & & & & 1 \\
\hline & 2 & & 1 & & & & 3 & 3 & 5 & 5 & 15 & 16 & 4 & 54 \\
\hline & & & 1 & & & & & & 1 & & & & 2 & 4 \\
\hline & 1 & & & & & & 4 & 1 & 2 & & 3 & 4 & 1 & 16 \\
\hline & 4 & & & 2 & & 2 & 3 & 8 & 13 & 6 & 7 & 7 & 3 & 55 \\
\hline & & & & & & & & & & & & & 1 & 1 \\
\hline & & & & & & 1 & & & 1 & & 1 & 3 & 1 & 7 \\
\hline & & & & & & & & & & & 1 & & & 1 \\
\hline & & & & & & & & & & & 2 & 2 & 2 & 6 \\
\hline & & & & & & & & & & & 1 & 2 & & 3 \\
\hline & & & & 1 & & & & 3 & 6 & 7 & 16 & 11 & 5 & 49 \\
\hline & & & & & & & & & & & & & 1 & 1 \\
\hline Total & 18 & 1 & 5 & 4 & 0 & 6 & 16 & 20 & 54 & 44 & 89 & 146 & 69 & 472 \\
\hline Percent * & 3.8 & 0.2 & 1.1 & 0.8 & 0.0 & 1.3 & 3.4 & 4.2 & 11.4 & 9.3 & 18.9 & 30.9 & 14.6 & 100.0 \\
\hline $\begin{array}{c}\text { Cumulative } \\
\text { Total }\end{array}$ & 18 & 19 & 24 & 28 & 28 & 34 & 50 & 70 & 124 & 168 & 257 & 403 & 472 & - \\
\hline $\begin{array}{l}\text { Cumulative } \\
\text { Percent** }\end{array}$ & 3.8 & 4.0 & 5.1 & 5.9 & 5.9 & 7.2 & 10.6 & 14.8 & 26.3 & 35.6 & 54.4 & 85.4 & 100.0 & - \\
\hline
\end{tabular}

*Minor differences between the "percent" row and the "cumulative percent" row are due to rounding error 


\section{Table 4: Net Growth of Settlements by Decade since 1900}

\begin{tabular}{cccccc} 
Time Period & $\begin{array}{c}\text { Founded during, } \\
\text { Existing Today }\end{array}$ & $\begin{array}{c}\text { Founded during, } \\
\text { Extinct Today }\end{array}$ & $\begin{array}{c}\text { Total } \\
\text { Founded }\end{array}$ & $\begin{array}{c}\text { Extinct } \\
\text { during }\end{array}$ & $\begin{array}{c}\text { Net Gain } \\
\text { / Loss }\end{array}$ \\
\hline $1900-1909$ & 1 & 15 & 16 & 10 & +6 \\
$1910-1919$ & 5 & 11 & 16 & 15 & +1 \\
$1920-1929$ & 4 & 11 & 15 & 12 & +3 \\
$1930-1939$ & 0 & 4 & 4 & 13 & -9 \\
$1940-1949$ & 6 & 8 & 14 & 9 & +5 \\
$1950-1959$ & 16 & 10 & 26 & 10 & +16 \\
$1960-1969$ & 20 & 21 & 41 & 14 & +27 \\
$1970-1979$ & 54 & 19 & 73 & 16 & +57 \\
$1980-1989$ & 44 & 24 & 68 & 14 & +54 \\
$1990-1999$ & 89 & 36 & 125 & 35 & +90 \\
$2000-2009$ & 146 & 11 & 157 & 36 & +121 \\
$2010-p r e s e n t$ & 69 & 2 & 71 & 15 & +56 \\
(as of 9/30/13) & 69 & 171 & 625 & 198 & 427 \\
\hline Total & 454 & 13 & & & -
\end{tabular}

Prior to $1900^{*} \quad 18 \quad 27$

*Eighteen is the number of settlements founded prior to 1900 that still exist today. Twenty-seven is the number of settlements founded prior to the twentieth century that became extinct anytime after 1900 .

\section{Table 5: List of Recently Extinct Settlements}

Ebensburg / Nicktown (Cambria County), Pennsylvania, 1997-2013

Princeton (Lawrence County), Pennsylvania, 2010-2011

Worthington (Greene County), Indiana 1993-2012

Tyrone (Schuyler County), New York, 2010-2012

Townville (Crawford County), Pennsylvania, 1972-2012

Vallonia (Jackson County), Indiana, 1998-2011

Centertown (Ohio County), Kentucky, 2007-2011

Salem (Livingston County), Kentucky,1993-2011

Coral (Montcalm County), Michigan, 2008-2011

Ludington (Mason County), Michigan, 1981-2011

Plain City, Madison County, Ohio, 1896-2011

Kirksville / Gibbs (Adair County), Missouri, 2000-2010

Wheatland (Hickory County), Missouri, 1994-2010

Whitehall (Jefferson County), Montana, 2001-2010

Beallsville (Monroe County), Ohio, 2007-2010

Mt. Vernon (Jefferson County), Illinois, 1987-2009 


\section{Table 6: Fifteen Amish Settlement Facts}

1. The first Amish settlement directory was published in the December 1974 issue of Family Life. It listed 112 settlements and 444 church districts. There are now over 4.2 times as many settlements $(472)$ and 4.5 times $(2,012)$ as many church districts as then (Table 1 ).

2. The oldest existing settlement is Lancaster/Chester Counties, PA, founded ca. 1760. Pennsylvania is the only state with settlements still existing today that were founded in the 1700s. Indiana has more settlements founded during the 1800s than any other state (Table 1).

3. The largest settlement is spread across five counties of northeast Ohio, including Holmes, Wayne, Tuscarawas, Coshocton, and Stark (Table 1).

4. At the present time, the Rexford, Montana, settlement is both the northernmost and the westernmost settlement. The southernmost settlement is Pinecraft, Florida, and the easternmost settlement is Fort Fairfield, Maine.

5. Since 2008, new states to host Amish settlements for the first time ever include South Dakota and Wyoming (Table 1).

6. There are many Amish settlements that straddle a county line but only ten which are multi-state. These include Berne / Monroe / Geneva (IN \& OH), Hamilton (IN \& OH), Seymour (IA \& MO), Lamoni / Davis City (IA \& MO), Granger / Cresco (MN \& IA), Mercer (MO \& IA), Kinsman ( $\mathrm{OH} \& \mathrm{PA})$, Pierpont / Conneaut ( $\mathrm{OH} \&$ PA), Meyersdale / Springs (PA \& MD), and Sugar Grove (PA \& NY). There are several other settlements that may have one or two households on the other side of a state line which are not on this list (Table 1).

7. There are five states with more than 100 church districts. These include Ohio, Pennsylvania, Indiana, Wisconsin, and New York (Table 2).

8. There are now four settlements with 100 or more church districts: Greater Holmes County, OH; Lancaster / Chester Counties, PA; Elkhart-LaGrange-Noble Counties, IN; and Greater Geauga County, OH (Table 1).

9. In 1974, 69 of the 112 settlements contained only one church district. In 2008, 208 of the 402 settlements contained one church district. There are now 245 settlements with one church district, 90 settlements with two church districts, 110 with three to nine church districts, and 27 settlements, including the four largest, with at least ten church districts. These 27 settlements can be found in 12 different states (Table 1).
10. There are 11 states and the province of Ontario that contain at least ten Amish settlements. Pennsylvania is the leader with 55, followed by Ohio (54), New York (50), and Wisconsin (49) (Table 2).

11. The county containing the most Amish settlements is Crawford (PA). Eight settlements are completely or partially located there, including one multi-state settlement. Mercer County, which is the next county south in Pennsylvania, contains all or part of seven settlements, also with one multi-state community. Plus, it shares one settlement with Crawford County. Adjoining Crawford County to the west is Ashtabula County, $\mathrm{OH}$, which includes all or part of seven settlements, sharing Pierpont/Conneaut with Crawford County, PA. Altogether, these three counties are the home to 19 distinct settlements. Localities playing host to six settlements (completely or partially) are Knox County, OH, Osceola County, MI, and Clark County, WI (Table 1). ${ }^{3}$

12. Only $7.2 \%$ of existing settlements were founded before 1950 , and $28.3 \%$ began sometime during the next four decades. Nearly one in five (18.9\%) were established in the 1990s, 30.9\% during 2000 to 2009, and $14.6 \%$ since January 1, 2010 (Table 3).

13. Every decade, beginning with the 1940s, has witnessed a net increase in Amish settlements. That is seven consecutive decades (Table 4)!

14. Of the 80 existing settlements founded since 2009, 30 are located in counties where there has never been a settlement before, and 50 in counties that already contained a settlement, or one was previously there which became extinct at least several years before the next settlement was established. Hence, it is not a replacement settlement, which are new settlements established about a year after the previous one becomes extinct (Table 1). ${ }^{4}$

15. Accounting for all settlements, both those founded and extinct during the past 23 years and nine months, there is a net gain of one new settlement every 4.64 weeks. This is a conservative estimate because the net gain of settlements (Table 4) was higher in this century than during the 1990s. Nonetheless, a net gain of one per every 4.64 weeks means a projected number of settlements at about 542 by 2020 , and nearly 900 by $2050 .^{5}$ 
Figure 1: Existing Settlements as of September 20, 2013

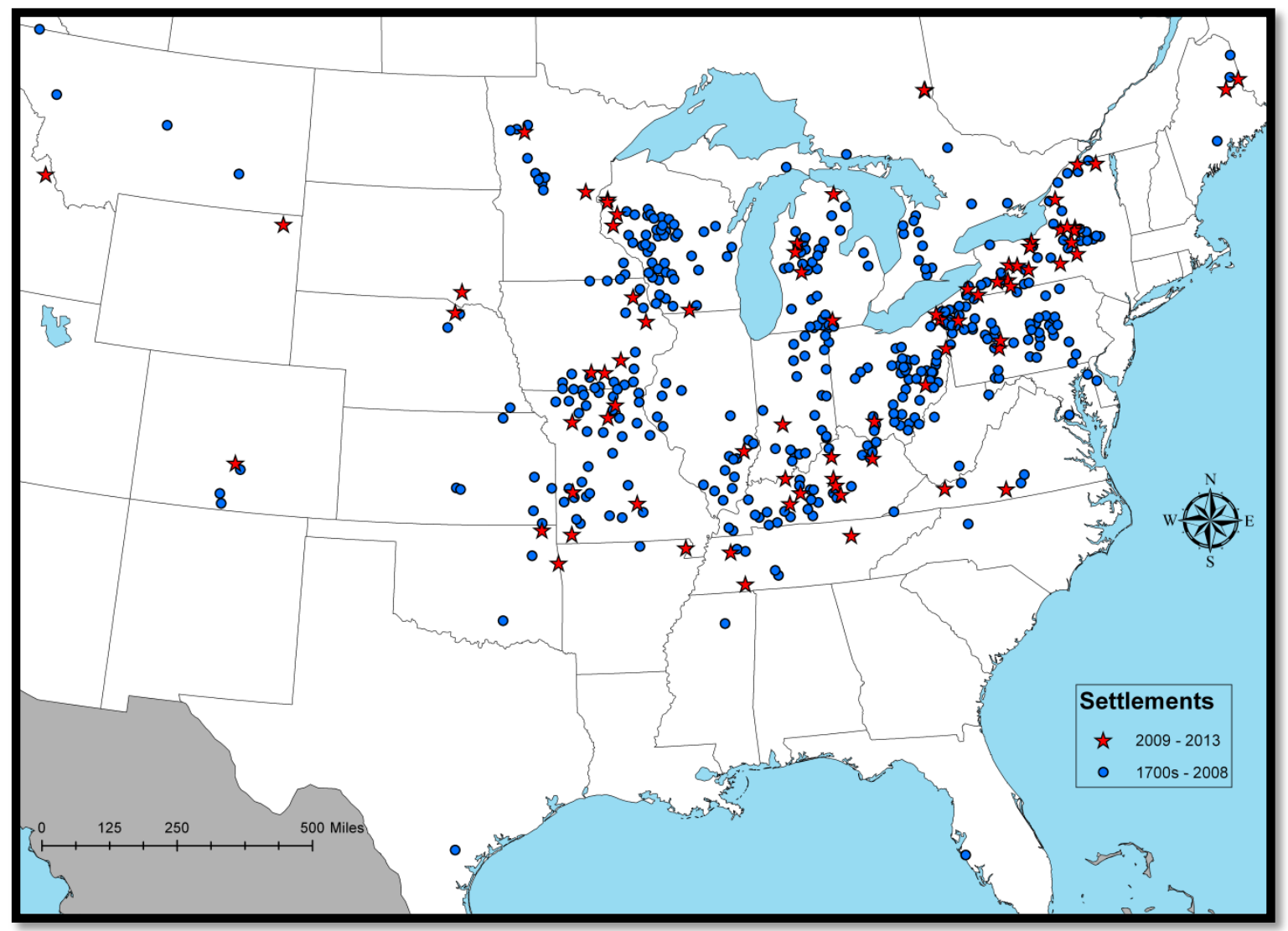

\section{Endnotes}

${ }^{1}$ Contact information: Joseph F. Donnermeyer, School of Environment and Natural Resources, Room 408C, Kottman Hall, 2021 Coffey Road, The Ohio State University, Columbus, Ohio 43210. 614292 9167; donnermeyer.1@osu.edu

${ }^{2}$ Waldrep $(2008,413)$ describes para-Amish as groups who have:

“...maintained a set of doctrinal positions and material standards, including the horse-and buggy, that closely parallel those of the Old Order Amish. In each case, though, differences of emphasis, practice, leadership, or setting precluded their being embraced by any other Amish group.”

${ }^{3} \mathrm{~A}$ list of the "Twenty-Five Most Amish Counties" can be found in the first issue of JAPAS (see Donnermeyer, Anderson \& Cooksey, 2013, Table 8). It shows Vernon County, Wisconsin, with six settlements; however, one (LaValle) was almost completely in Sauk County, with only a small population in Vernon. We did not include this in our current considerations. There are a 
number of other settlements where a small percentage lives in an adjoining county, but too small to be considered significant.

${ }^{4}$ Discerning readers may notice that the numbers do not quite add up to a net gain of 70 settlements as stated in the first paragraph of this article. The reason is simple: as more information becomes available, what was listed as a settlement previously was not ever one because a third family did not move there, or we subsequently discovered that what looked like a new settlement was actually a few families near the edge of an established community who had a different address and postal code. Although we strive for accuracy, we recognize that mistakes can be made. When discovered, they are corrected.

${ }^{5} \mathrm{~A}$ similar set of projections were made in the first issue of JAPAS by Donnermeyer, Anderson and Cooksey (2013) (see Table 9). These projections represent settlement growth adjusted for the years since 2010. The differences between the estimates in that article and here are miniscule when considered in the context that settlement growth, unless other factors intervene, will remain high for the foreseeable future.

\section{References}

Donnermeyer, Joseph F., Cory Anderson, and Elizabeth Cooksey. 2013. “The Amish Population: County Estimates and Settlement Patterns.” Journal of Amish and Plain Anabaptist Studies 1(1):72-109.

Donnermeyer, Joseph F. and Elizabeth C. Cooksey. 2010. "On the Recent Growth of New Amish Settlements.” The Mennonite Quarterly Review 84(2):87-116.

Luthy, David. 2009. Amish Settlements across America: 2008. Aylmer, ON: Pathway Publishers.

Luthy, David. 2012. Why Some Amish Communities Fail: Extinct Settlements, 1961-2011. Aylmer, ON: Pathway Publishers.

Raber, Aden B. 2012. The New American Almanac. Baltic, OH: Raber’s Book Store.

Waldrep, G.C. 2008. “The New Order Amish and Para-Amish Groups: Spiritual Renewal within Tradition.” The Mennonite Quarterly Review 82(3):395-426. 